\title{
Double photoproduction of neutral pions on light nuclei in coherent reactions
}

\author{
Mikhail Egorov*广 \\ National Research Tomsk polytechnic university \\ E-mail: egorovphys@mail.ru
}

We discuss photoproduction of $\pi^{0} \pi^{0}$ pairs on light nuclei with A-number increase. We focus mainly on the $\left(\gamma, \pi^{0} \pi^{0}\right)$ reaction details like as couplings list that was chosen in accordance with PDG-2014 predictions, nuclear transition matrix element and final state interaction problem. Total cross sections for the proton, deuteron, ${ }^{3} \mathrm{He},{ }^{7} \mathrm{Li}$ and ${ }^{12} \mathrm{C}$ are presented. As the original part of the work final $\pi \pi$ rescattering is introduced vie $I=0, S=0$ mesonic resonance.

XXII International Baldin Seminar on High Energy Physics Problems,

15-20 September 2014

JINR, Dubna, Russia

\footnotetext{
* Speaker.

$\dagger$ The author acknowledge suppot from the Dynasty foundation, the TPU grant LRU-FTI-123-2014 and the MSU program 'Nauka' (project 825). M. Egorov also appreciates his father V. Egorov for the all over furtherance.
} 


\section{Introduction}

Reactions of pion photoproduction are known to be a powerful tool in studying nucleon excitation spectrum. Due to the weak coupling of initial photons with neutral pion in reaction $\left(\gamma, \pi^{0}\right)$ a sizable contributions like as meson pole term and $\Delta$-Kroll-Ruderman term are strongly suppressed. Therefore the relative role of final state interaction (FSI) in total cross section $\left(\gamma, \pi^{0}\right)$ is considerable enough (see for example [1]). Besides, some of the predicted by QCD nucleon resonances are invisible [2] in single photoproduction channel. That is way reactions of double pion photoproduction are seem to be a fine tool in searching the nucleonic excitations invisible in single photoproduction.

We concentrate on coherent reactions $\left(\gamma, \pi^{0} \pi^{0}\right)$ only where isospin of the target is zero (deuteron, ${ }^{12} \mathrm{C}$ ) or is equal to $1 / 2$ (proton, ${ }^{3} \mathrm{He},{ }^{7} \mathrm{Li}$ ). Role of nuclear p-shell model is also discussed in reaction on ${ }^{7} \mathrm{Li}$ and ${ }^{12} \mathrm{C}$ as a target. Two pion rescattering process according to the works [3, 4] where this effect on the one hand is considerable enough in threshold region of the reaction $\left(\gamma, \pi^{0} \pi^{0}\right)$ and dislocates the maximum in $d \sigma / d \omega_{\pi \pi}$-distribution to a small mass region with A-number increase on the other is also included via effective $\sigma$-mesonic resonance.

\section{Model ingredients}

In this section, we briefly discuss the photon induced mechanism of the coherent process

$$
\gamma\left(\vec{k}, \varepsilon_{\lambda}\right)+A\left(\vec{p}_{i}, E_{A}\right) \rightarrow \pi^{0}\left(\vec{q}_{\pi_{1}}, E_{\pi_{1}}\right)+\pi^{0}\left(\vec{q}_{\pi_{2}}, E_{\pi_{2}}\right)+A\left(\vec{p}_{f}, E_{A^{\prime}}\right),
$$

where 4-momenta of participating particles are given in parenthesis and photon polarization vector is $\varepsilon_{\lambda}$, with index $\lambda= \pm 1$. Total unpolarized cross section for the process (2.1) has the form

$$
\frac{d \sigma}{d \Omega_{p_{f}} d \Omega_{q_{\pi}^{*}} d \omega_{\pi \pi}}=\frac{E_{A} E_{A^{\prime}} q_{\pi}^{*} p_{f} \alpha}{(2 \pi)^{5} 16 E_{\gamma} W^{2}(2 J+1)} \sum_{\lambda, M_{f}, M_{i}}\left|T_{M_{f}, M_{i}}^{\lambda}\right|^{2}
$$

In equation (2.2) $W$ is total energy in center-of-mass (c.m.) frame of the process (2.1); $p_{f}-$ is momentum of nucleus $A^{\prime}$ and corresponding solid angle is $\Omega_{p_{f}}$; photon energy in c.m. and invariant mass of two pions is given by $E_{\gamma}$ and $\omega_{\pi \pi}$ correspondingly; momentum of one pion in $\pi \pi$ center of mass system and the corresponding solid angle are given by $q_{\pi}^{*}$ and $\Omega_{q_{\pi}^{*}}$; total angular momentum of nucleus $A$ is $J ; \alpha$ is taken into account from identity of the pions and has the value $1 / 2$. An approach how to calculate matrix elements $T_{M_{f}, M_{i}}^{\lambda}$ under the relativized isobar model was proposed some time ago for example in Ref.[5]. The leading order contributions to cross section (2.2) are taken the place from $N-, \Delta-$ Born terms and resonance contributions that have been calculated under tree level. The final two-pions state in resonance induced terms then results from a two-step decay via intermediate quasi-two-body channels for which we take $\pi-\Delta$ and $\sigma-N$. Single body operator under the subject matter can then be written as

$$
\hat{T}_{\gamma N}^{\lambda}=\hat{T}_{\gamma N}^{B, \lambda}+\hat{T}_{\gamma N}^{\pi \Delta, \lambda}+\hat{T}_{\gamma N}^{\sigma N, \lambda}
$$

for the Born $-\hat{T}_{\gamma N}^{B, \lambda}$ and resonance terms $\hat{T}_{\gamma N}^{\pi \Delta, \lambda}$ and $\hat{T}_{\gamma N}^{\sigma N, \lambda}$. It should be noted that for the electromagnetic and hadron decay widths we used coupling list presented, for example in Ref.[6], 
that results from PDG-2014 [7] predictions by average extracted widths. Corresponding contributions to the proton spectra in reaction $p\left(\gamma, \pi^{0} \pi^{0}\right) p$ are illustrated on Fig.(1). According to the [7] predictions a number of nucleon resonances that originally excluded in Ref.[5] should be extended into $\Delta(1600) P_{33}^{\star \star \star}, N(1700) D_{13}, N(1650) S_{11}, \Delta(1905) F_{35}, \Delta(1910) P_{31}, N(1720) P_{13}$ since new information about their decay widths has appeared. Label a.c. on Fig.(1) just signs additional resonance contributions in accordance with PDG-2014 [7] information. As the original part of
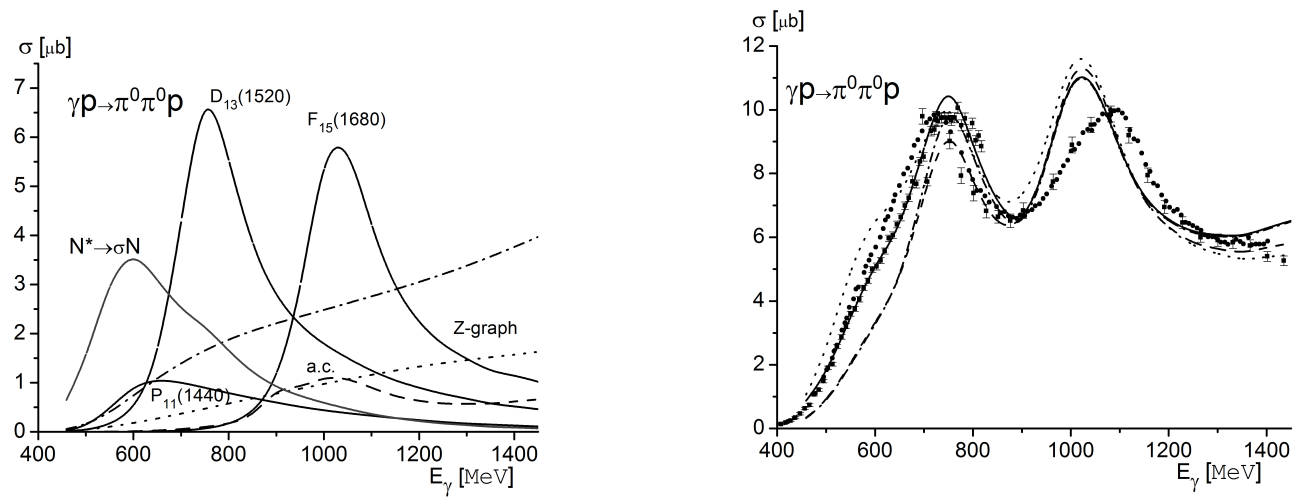

Figure 1: Left panel: spectrum of proton excitation; $N^{*}-$ corresponds to $S_{11}(1535), S_{11}(1650), F_{15}(1680), D_{13}(1520), P_{11}(1440)$ resonances; a.c. - is deciphered in the text. Right panel: total cross section of the process $p\left(\gamma, \pi^{0} \pi^{0}\right) p$. Dotted - predictions with $f_{\sigma \pi \pi}=3.76[5]$ without a.c.; dashed (dash-dotted) curve - inclusion fitted $f_{\sigma \pi \pi}$ coupling [6] without (with) a.c.; solid line corresponds to $\pi \pi$-rescattering effect with fitted $f_{\sigma \pi \pi}$ coupling and with a.c. Data: circles [11], squares [12].

the work rescattering mechanism $\pi^{+} \pi^{-} \rightarrow \pi^{0} \pi^{0}$, where intermediate mesonic resonance with zero spin and zero isospin $(I=0, S=0)$ in $s$-partial wave, is included due to effective $\sigma$-meson exchange. Phase shifts of $\pi \pi$ rescattering were fairly well fitted by an appropriate coupling $f_{\sigma \pi \pi}$ choice where sigma mass has the value $m_{\sigma}=700 \mathrm{MeV}$ and sigma width was parametrized by $\Gamma_{\sigma}=\sqrt{\omega_{\pi \pi}^{2} / 4-m_{\sigma}^{2}} \mathrm{MeV}[6]$.

Processes where mass number $A \leq 2$ were considered in the following manner. Deuteron wave function was chosen from Ref.[8] (energy dependent solution) wherein deuteron form factor is well described up to $E_{\gamma}=700 \mathrm{MeV}$. Wave functions of ${ }^{3} \mathrm{He}$ was also taken into account under the Ref.[9] where its behavior is reasonable enough in a wide range of momentum transfer. In $p$-shell nuclei $\left({ }^{7} \mathrm{Li}\right.$ and $\left.{ }^{12} \mathrm{C}\right)$ in order to calculate nuclear matrix element we use shell model with intermediate couplings where $p$-shell nucleon's momentum distribution had to be taken into account from Ref.[10].

\section{Results and discussion}

Here we have shown total cross sections on proton, deuteron, ${ }^{3} \mathrm{He},{ }^{7} \mathrm{Li}$ and ${ }^{12} \mathrm{C}$. We proposed an approach where the relativized isobar model (for example [5]) was extended by a number or resonance terms are known to be of noticeable contributions in $\pi^{0} \pi^{0}$ channel nowadays. All of 
them as it is seen on Fig.(1) are of small magnitude. It is against this background that using fitted coupling $f_{\sigma \pi \pi}$ in photoproduction operator (2.3) dramatically changes predictions of proton cross section in second resonance region. Inclusion of rescattering term according to the Ref.[6] enhances the prediction up to $E_{\gamma}=850 \mathrm{MeV}$. In reactions where mass number $A=2$ and $A=3$ we have
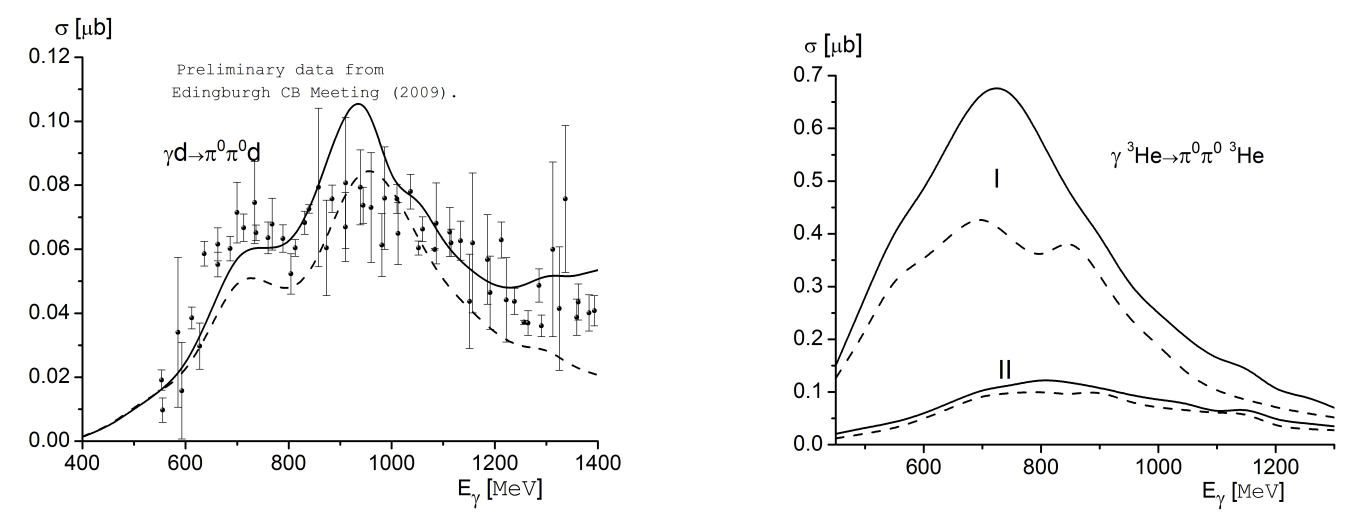

Figure 2: Left panel: total cross section of the $d\left(\gamma, \pi^{0} \pi^{0}\right) d$ reaction; dashed line - calculations without a.c.; solid - all terms. Preliminary data from [13]. Right panel: total cross section of the ${ }^{3} \mathrm{He}\left(\gamma, \pi^{0} \pi^{0}\right)^{3} \mathrm{He}$ reaction; dashed (solid) line - prediction without (with) $s$-wave $\pi \pi$ rescattering with a.c. in all cases; (I) and (II) labels correspond to simplified and exact solution.

following situation. In deuteron case, where resonances with isospin $T=1 / 2$ contribute to (2.3) only ( $\pi \pi$ rescattering term is also forbidden), a.c. terms are of visible meaning in a wide photon energy range. In helium-3 where initial and final nucleus has isospin $1 / 2$ two solutions (I and II) were done and presented on right panel of Fig.(2). First one (I) corresponds situation where nuclear transition matrix element is merely multiplied by helium wave function $\Psi^{v}$ where $v=1,2,3,4,5$ [9] leaving out of account relationships between partial waves and other quantum numbers under the impulse approximation framework. It is second solution (II) where nuclear matrix element was exactly calculated for all of $v$-terms separately under the impulse approximation framework.

Nuclear matrix element in p-shell nuclei was calculated vie to step the former of that is the ground state only the later is ground state with first excited state. In lithium-7 as the ground state we use $[3]^{22} P$ and the most probable nucleon excited state we use $[21]^{24} P$ where in brackets []-Young tableau and $P$ is nucleon orbital momentum (for spin and isospin we use following notation ${ }^{2 T+12 S+1} L$ ). The same procedure was done for ${ }^{12} C$ where ground and first excited state was treated due to $\left.[44]]^{11} S,[431]\right]^{13} P,[422]{ }^{15} D$. Final state interaction of pions and nucleus ${ }^{12} C$ is introduced vie simple potential model [14] with optical potential from Ref.[15]. Standard multiple inelastic $\pi-N$ rescattering is treated throughout meson-hadron coupling constants. As the matter of fact and as it is evident from Ref.[6] same procedure is fairly well reproduces cross section of the $d\left(\gamma, \pi^{0}\right) d$ process.

Resulting cross section for all of considered here p-shell nuclei is shown on Fig.(3). Role of fist excited states is of great importance on ${ }^{12} C$ where cross section is seems to be doubling (dotted lines). Less important but also of visible size first excited state is seen on ${ }^{7} \mathrm{Li}$ (dotted lines). Contribution of $s$-wave $\pi \pi$ rescattering is seems to be of great magnitude near $E_{\gamma}=550 \mathrm{MeV}$. 

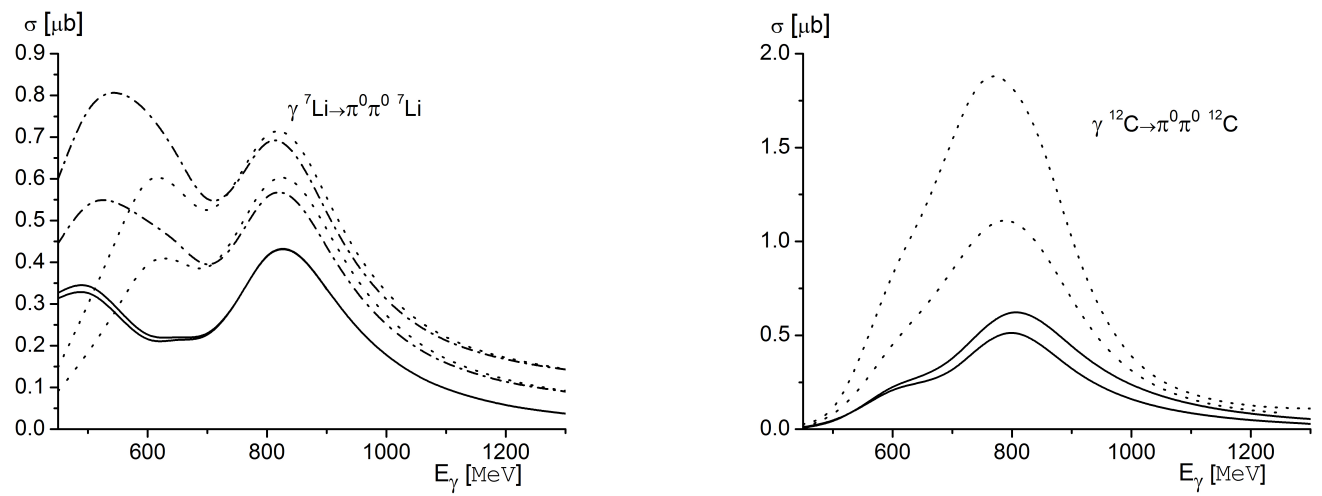

Figure 3: Dotted lines - plane wave calculations; dash-dotted lines - inclusion $s-$ wave $\pi \pi$ rescattering; solid lines - distort wave impulse approximation [14]. Left panel: total cross section of the ${ }^{7} \operatorname{Li}\left(\gamma, \pi^{0} \pi^{0}\right)^{7} \mathrm{Li}$ reaction; the difference between duplicated lines is that fist most probable excited level is included in nuclear matrix element. Right panel: total cross section of the ${ }^{12} C\left(\gamma, \pi^{0} \pi^{0}\right){ }^{12} C$ reaction; the difference between duplicated lines is that three most probable excited level is included in nuclear matrix element.

It is interesting to note the maximum of the rescattering effect under consideration is shifted to the small photon energy region from proton to lithium-7. Damping cross section that has been originated under potential model used to check pion-nucleus interaction in final state brings an ambiguousness. Later is that plane wave predictions with and without excited state inclusion more deffer among themselves than distort wave impulse approximations.

\section{Conclusions}

Here we have shown total cross section of the process $\left(\gamma, \pi^{0} \pi^{0}\right)$ on proton, deuteron, ${ }^{3} \mathrm{He},{ }^{7} \mathrm{Li}$ and ${ }^{12} C$. Photoproduction operator was calculated according to the nowadays information about nucleon excitation spectrum [7]. For the simplicity we was included only $s-$ wave $\pi \pi$ rescattering in final state where we had been achieved a noticeable effect. We leave the investigation of inelastic pion-nucleus interaction and more complex $p$-wave $\pi \pi$ rescattering to future studies.

\section{References}

[1] J. M. Laget, Phys. Lett.B 68 (1997) 58.

[2] L. A. Copley, G. Karl, E. Obryk, Nucl. Phys. B 13 (1969) 303;

R. Koniuk, N. Isgur, Phys. Phys. D 21 (1980) 1868.

[3] E. Oset, L. Roca, M. J. Vicente Vacas, J. C. Nacher, in: International Workshop on Chiral Fluctuations in Hadronic Matter, Orsay, France. 26-28 Sep. (2001);

G. Messchendorp [et al.], Phys, Rev. Lett. 89 (2002) 222302.

[4] L. Roca, E. Oset, M. J. Vicente Vacas, Phys. Lett. B 541 (2002) 77;

L. Roca, E. Oset, M. J. Vicente Vacas, Nucl. Phys. A 721 (2003) 719.

[5] A. Fix, H. Arenhövel, Eur. Phys. J. A 25 (2005) 115. 
[6] M. Egorov, Physics of Atomic nuclei (2015) (to be published);

M. Egorov, Dissertation for Candidate of Physical and Mathematical Sciences (2015).

[7] K. A. Olive [et al.], (Particle Data Groupe), Chin. Phys. C 38 (2014) 090001.

[8] R. Machleidt, K. Holinde, C. Elster, Phys. Rep. 149 (1987) 1.

[9] V. Baru, J. Haidenbauer, C. Hanhart, J. A. Niskanen, Eur. Phys. J. A 16 (2003) 437.

[10] D. Drechsel, L. Tiator, S. Kamalov, S. Yang, Nucl. Phys. A 660 (1999) 423.

[11] V. Kashevarov [et al.], Phys. Rev. C 85 (2012) 064610.

[12] Y. Assafiri [et al.], Phys. Rev. Lett. 90 (2003) 222001.

[13] I. Jaeglé, CB meeting, Edinburgh, Sep. 2009.

[14] V. Tartakovski, I. Kozlovski, E. Maljarj, Ukrainian Physical Journal 21 (1976) 1823; 23 (1978) 368; V. Balashov, D. Meboniya, Proceedings of NAS RA - Physics 3 (1968) 122; 3 (1968) 167.

[15] K. Seth, Nucl. Phys. A 138 (1969) 61. 